\title{
Examining Psychometric Characteristics of a Menopausal Health Questionnaire: Translation and Psychometric Evaluation of the Persian Version
}

\author{
Nasibeh Sharifi ${ }^{1}$, Somayyeh Khazaeian², Safoura Khazaeian ${ }^{3}$, Marzieh Masjoudi ${ }^{4}$, Azita Fathnejad Kazemi ${ }^{5}$, \\ Anvar-sadat Nayebi nia ${ }^{6}$
}

${ }^{1}$ M.Sc. of Midwifery, Ph.D. Candidate of Reproductive Health, Student Research Committee, School of Nursing and Midwifery, Shahid Beheshti University of Medical Sciences, Tehran, Iran

${ }^{2}$ M.Sc. of Midwifery, Pregnancy Health Research Center, School of Nursing and Midwifery, Zahedan University of Medical Sciences, Zahedan, Iran

${ }^{3}$ Gynecologist, Department of Obstetrics and Gynecology, Zahedan University of Medical Sciences, Zahedan, Iran

${ }^{4}$ M.Sc. of Midwifery, Faculty Member, Department of Midwifery, School of Nursing and Midwifery, Islamic Azad University of Rasht, Rasht, Iran

${ }^{5}$ M.Sc. of Midwifery, Faculty Member, Department of Midwifery, School of Nursing and Midwifery, Islamic Azad University of Tabriz, Tabriz, Iran

${ }^{6}$ M.Sc. of Midwifery, Faculty Member, Department of Midwifery, School of Nursing and Midwifery, Islamic Azad University of Karaj, Karaj, Iran

\section{Type of article: Original}

\begin{abstract}
Introduction: Menopause is a natural event in a woman's life which affects her general health and quality of life. However, currently there is no Persian instrument for measuring health status during this period. Therefore, the present study was performed to assess the validity and reliability of the Persian version of the Menopausal Health Questionnaire in Iran.

Methods: The questionnaire was translated to Persian with the approach of Jones et al and its validity was assessed by a panel of experts. The Persian version was tested among 300 patients who had referred to the gynecological clinic at the Ali ibn Abi Talib Hospital in Zahedan. Convenience method was used for sampling. Exploratory factor analysis was used to assess construct validity of collected data. Test-retest method was used to determine reliability in two-week intervals and Cronbach's alpha coefficient was used to check the internal consistency.

Results: According to research findings, the exploratory factor analysis showed an acceptable fitness. Varimax rotation indicated 6 factors with Eagan values more than 1, which explained $49.27 \%$ of the variance. Cronbach's alpha coefficient for the entire tool was 0.84 and it was from 0.50 to 0.82 for the subcategories. All factors had a significant case-total correlation. Results of test-retest showed the stability of the questionnaire and its subcategories, and the intraclass correlation coefficient (ICC) was assessed to be 0.93 .

Conclusions: The 14-item Menopausal Health Questionnaire is a valid and reliable instrument to assess postmenopausal women's health.
\end{abstract}

Keywords: Health, Menopause, Psychometrics, Factor Analysis

\section{Introduction}

Promoting health level and enjoying acceptable health improves women's quality of life in different stages of life, and provides significant benefits for the community. Menopause is a sensitive period in a woman's life in which, a woman enters a new phase of life with the passage of the reproductive cycle (1). All women experience menopause with aging, and with this experience, they are faced with extensive changes (2). Most of these changes are hormone-

\section{Corresponding author:}

Somayyeh Khazaeian, Zahedan University of Medical Sciences, Zahedan, Iran.

Tel: +98.9151430266, Email: m_khazaiyan@yahoo.com

Received: July 18, 2016, Accepted: November 16, 2016, Published: January 2017

iThenticate screening: October 02, 2016, English editing: December 20, 2016, Quality control: January 02, 2017

(C) 2017 The Authors. This is an open access article under the terms of the Creative Commons Attribution-NonCommercialNoDerivs License, which permits use and distribution in any medium, provided the original work is properly cited, the use is non-commercial and no modifications or adaptations are made. 
dependent and associated with reducing estrogen levels. The most common symptoms are related to the vasomotor system including hot flashes and night sweats; other symptoms include dizziness, rapid and irregular heart rate, vaginal mucosa atrophy and bladder irritability, mood swings, sleep disturbances, headache, muscle aches, joint pain, and difficulty in concentration and memory disorders $(2,3)$. In addition to hormonal changes, the aging process also creates some problems, including muscle atrophy and changes in the status and structure of the skeleton (4). Problems caused by menopausal changes affect women's health condition by causing them tension and significant disability, which can impose additional pressure on the health care system (5). Due to increased life expectancy, more than a third of a woman's life is spent in post-menopausal period $(3,4)$. So, taking basic measures to promote post-menopausal women's health, seems essential, because, considering health conditions and its promoting methods among this group of women, it can affect the quality of life of the remaining years, and allow more fulfillment in the final third of their lives. (6-8). Iranian women are no exception. Necessity of having knowledge as a first step for planning on one side, and the lack of appropriate instruments for measuring the health status of Iranian postmenopausal women on the other side lead to researchers making an effort in finding an instrument among those available, in which to measure different aspects of health. In this regard, it was essential to assess validity and reliability, and the standardization of a questionnaire more suited to the conditions of the country. Hence, this study was aimed at determining the psychometric characteristics of the Menopause Health Questionnaire in order to localize a comprehensive questionnaire for identifying factors affecting postmenopausal women's health in Iran, and take a small step towards improving women's health and quality of life.

\section{Material and Methods}

\subsection{Menopause Health Questionnaire}

The Menopause Health Questionnaire was first developed and published by the America Menopause Society in 2005. The scale consists of fourteen sections including demographic data, referral reason, anthropometric data (height and weight), medical history, history of major illnesses or injuries, gynecologic history (last menstrual period, type of menopause, mammograms, vaginal douching, etc.), midwifery history (contraception methods, number of pregnancies, deliveries, etc.), sexual history, allergic information, drug history (hormone therapy, medication or supplement), family history of diseases, personal habits (exercise, diet, smoking, caffeine, alcohol, abuse, and stress management), menopause system and individuals' insights about menopause and hormone therapy.

\subsection{Translation of instrument to Persian}

The research instrument was translated to Persian, after the permission of the designer, with the approach of Jones et al, which was a mixture of symmetric and asymmetric strategies (9). For translation, we assed several stages including the following:

1) The use of bilingual translators who were familiar with both cultures;

2) Translation from English to Persian by two translators, and from English into Farsi by two separate translators;

3) Experts' evaluation of the original English and Persian versions to evaluate clarity, differences and errors in meaning construction. The composition of the expert panel consisted of 3 reproductive health experts, two obstetrician specialists, one psychometric expert, and three postmenopausal women;

4) Fixing all differences in meaning construction between translation and the translated version;

5) Final version revision.

\subsection{Determining the sample size}

Based on a number of studies for factor analysis, the appropriate sample size was reported to be between 3 and 10 per statement of the instrument $(10,11)$. In this study, to estimate the sample size, the questionnaire was given to 300 samples. Kaiser-Mayer-Olkin test (KMO) and Bartlett's test were used to evaluate the adequacy of the data and the correlation matrix. Results showed that the KMO ratio was 0.704 and the Bartlett test (3316.19) showed that the sample size of 300 was enough.

\subsection{Assessing the construct validity}

A cross-sectional study was conducted to assess the construct validity of the developed instrument. Literate postmenopausal volunteer women were selected for this part of the study. The study population included individuals who had referred to the gynecological clinic in Ali ibn Abi Talib Hospital in Zahedan. For this purpose, after receiving required permission and written consent from the samples, simple sampling was conducted on 300 postmenopausal women who had referred to Ali ibn Abi Talib Hospital in Zahedan from June to December, 2015. 
Samples were assured of the confidentiality of their personal information. After completion of data gathering, all participants were given a pamphlet on the subject of menopause, its symptoms and how to deal with this period.

\subsection{Assessing the instrument's reliability}

To determine the questionnaire's reliability, Cronbach's alpha coefficient and intraclass correlation coefficient (ICC) were used. Thus, Cronbach's alpha was used for each factor, as well as the entire questionnaire, to provide the internal consistency of the questionnaire phrases, and the test-retest method was used to assess the stability. Researchers provided the instrument for 20 postmenopausal women in two stages with an interval of 2 weeks. This means that questionnaires were completed in the first step in the admission of women to the Ali ibn Abi Talib Hospital and in the second step two weeks later with previous coordination or phone call, and the obtained scores in these two stages were compared using intraclass correlation index (ICC). Data were analyzed using the SPSS software Ver.22.

\section{Results}

\subsection{General findings}

Findings showed that the mean age of the participants in the study was $56.83 \pm 6.16$ years (minimum age of 42 years and maximum age of 70 years). The mean number of pregnancies, number of children, age at first pregnancy and age at last birth were $6.02 \pm 2.97,5.26 \pm 2.37,18.06 \pm 5.37$ and $32.48 \pm 8.24$, respectively, and the mean height and weight of the samples were $1.59 \pm 7.21$ and $69.09 \pm 10.55$, respectively. Table 1 showed the frequency distribution of demographic characteristics of the study samples. Reasons for referring subjects to the hospital included 106 hot flashes cases (3.35\%), 82 cases of joint pain, (27.3\%), 31 cases of bleeding (10.3\%), 19 cases of urinary incontinence $(6.3 \%), 18$ cases of blood spotting $(6 \%), 17$ cases of vaginal dryness $(5.7 \%), 11$ cases of delayed menstruation (3.7\%), 11 cases of dysuria (3.7\%) and 5 cases of infection $(1.7 \%)$.

Table 1. Distribution of demographic characteristics

\begin{tabular}{|c|c|c|c|c|c|c|c|}
\hline \multicolumn{2}{|l|}{ Variable } & $\mathrm{n}$ & $\%$ & \multicolumn{2}{|l|}{ Variable } & $\mathrm{n}$ & $\%$ \\
\hline \multirow[t]{5}{*}{ Ethnic } & Persian & 190 & 63.3 & \multirow[t]{5}{*}{ Number of pregnancies } & 0 & 13 & 4.3 \\
\hline & Baluchis & 56 & 18.7 & & \multirow[t]{2}{*}{$<4$} & \multirow[t]{2}{*}{43} & \multirow[t]{2}{*}{14.3} \\
\hline & Turks & 7 & 2.3 & & & & \\
\hline & Kurds & 19 & 6.3 & & \multirow[t]{2}{*}{$\geq 4$} & \multirow[t]{2}{*}{244} & \multirow[t]{2}{*}{80.4} \\
\hline & Lurs & 28 & 9.3 & & & & \\
\hline \multirow[t]{5}{*}{ Marital status } & Single & 15 & 5 & \multirow[t]{5}{*}{ First-person support } & Husband & 144 & 48 \\
\hline & Married & 204 & 68 & & Daughter & 111 & 37 \\
\hline & \multirow[t]{2}{*}{ Divorced } & \multirow[t]{2}{*}{8} & \multirow[t]{2}{*}{2.7} & & Sister & 20 & 6.7 \\
\hline & & & & & Mother & 8 & 2.7 \\
\hline & Widowed & 73 & 24.3 & & Friend & 17 & 5.7 \\
\hline \multirow[t]{4}{*}{ Occupation } & Unemployed & 244 & 81.3 & \multirow[t]{4}{*}{ First Health Care Center } & Health center & 115 & 38.3 \\
\hline & Employed & 35 & 11.7 & & Midwifery office & 53 & 17.7 \\
\hline & \multirow[t]{2}{*}{ Retired } & \multirow[t]{2}{*}{21} & \multirow[t]{2}{*}{7} & & Women's clinic & 89 & 29.7 \\
\hline & & & & & Hospital & 43 & 14.3 \\
\hline
\end{tabular}

\subsection{Exploratory factor analysis}

Exploratory factor analysis was conducted on the thirteenth part of the questionnaire (menopausal symptoms) on all 300 completed questionnaires. The KMO Index value was 0.704 in the first output which suggested that the selected sample was sufficient for the exploratory factor analysis. Also, Bartlett's sphericity test showed the suitability of factor analysis to identify the structure of factor model at a significance level of $p<0 / 001$, and showed detectable relationships between the variables that were factor analyzed. The second output of exploratory factor analysis consisted of two parts, including a specific primary value and the specific value of the extracted factors with rotation. Results of the rotational correlation matrix table showed that the set of 32 items measured six factors and these 6 factors could explain $49.27 \%$ of the variance of the 32 items related to menopause symptoms. The variance of each 6 items were 18.8, 7.83, 6.87, 6.41, 4.89 and 4.45, respectively, and six factors were extracted and selected based on the criterion of eigenvalues greater than one, and slope of scree plot graph (Figure 1). The first factor consisted of nine items that their load factor varied from $0.389-0.741$. The second factor included 4 items with loadings between $0.601-0.688$, the third factor included 7 items with loadings from $0.352-0.722$, the fourth factor included 3 items with loadings between $0.655-0.786$, the fifth factor included 5 items with loadings from 0.437 - 
0.743 , and finally the sixth factor included 5 items with loadings between $0.353-0.671$. According to factor analysis, questions were classified in 6 domains (mood, urinary, sexual, genital, vasomotor and physical) and the factor loadings for the final 32-item model are presented in Table 2.

Table 2. The rotated matrix of factor analysis components of menopausal symptoms instrument

\begin{tabular}{|l|l|l|}
\hline Items of menopausal symptoms assessment instrument & Factor loading \\
\hline Factor 1: Mood & 1. I feel more tired than usual & 0.491 \\
& 2. I have difficulty concentrating & 0.596 \\
& 3. My memory is poor & 0.520 \\
& 4. I am more irritable than usual & 0.618 \\
& 5. I feel more anxious than usual & 0.534 \\
& 6. I have more depressed moods & 0.717 \\
& 7. I am having mood swings & 0.741 \\
& 8. I have crying spells & 0.693 \\
& 9. I have headaches & 0.389 \\
\hline Factor 2: Unitary & 10. I need to urinate more often than usual & 0.688 \\
& 11. I leak urine & 0.586 \\
& 12. I have pain and burning when urinating & 0.601 \\
& 13. I have bladder infections & 0.604 \\
\hline Factor 3: Sexual & 14. My vagina is dry & 0.502 \\
& 15. I have pain during intercourse & 0.632 \\
& 16. I have pain inside during intercourse & 0.772 \\
& 17. I have bleeding after intercourse & 0.391 \\
& 18. I lack desire or interest in sexual activity & 0.591 \\
& 19. I have difficulty achieving orgasm & 0.609 \\
& 20. My opportunity for sexual activity is limited & 0.352 \\
\hline Factor 4: Genital & 21. I have vaginal itching & 0.655 \\
& 22. I have an abnormal discharge & 0.733 \\
& 23. I have vaginal infections & 0.786 \\
\hline Factor 5: Vasomotor & 24. I have hot flashes & 0.681 \\
& 25. I have night sweats & 0.743 \\
& 26. I have difficulty getting to sleep & 0.474 \\
& 27. I have difficulty staying asleep & 0.520 \\
& 28. I get palpitations or sensation of butterflies in my chest and stomach & 0.437 \\
\hline Factor 6: Physical & 29. My stomach feels like it's bloated or I've gained weight & 0.353 \\
& 30. I have uncontrollable loss of stool or gas & 0.671 \\
& 31. I have breast tenderness & 0.473 \\
& 32. I have joint pains & 0.479 \\
\hline
\end{tabular}

\subsection{Reliability of data collection}

The internal consistency test method was used to assess the reliability of the instrument, and the calculated Cronbach's alpha values for whole and individual factors of the instrument were presented in Table 3 after conducting factor analysis. Cronbach's alpha coefficient of the entire questionnaire was calculated to be 0.84 and the intraclass correlation coefficient for the entire questionnaire was 0.935 .

Table 3. Correlation, Reliability and internal consistency evaluation questionnaires domains

\begin{tabular}{|l|l|l|l|l|}
\hline Factors & $\begin{array}{l}\text { Cronbach's } \\
\text { alpha }\end{array}$ & $\begin{array}{l}\text { Intraclass correlation } \\
\text { coefficient }\end{array}$ & $\begin{array}{l}\text { Confidence Interval (CI } \\
95 \%)\end{array}$ & $\begin{array}{l}\text { Number of } \\
\text { questions }\end{array}$ \\
\hline Factor 1: Mood & 0.82 & 0.954 & $0.919-0.979$ & 9 \\
\hline Factor 2: Unitary & 0.55 & 0.817 & $0.665-0.917$ & 4 \\
\hline Factor 3: Sexual & 0.70 & 0.930 & $0.874-0.967$ & 7 \\
\hline Factor 4: Genital & 0.71 & 0.889 & $0.792-0.950$ & 3 \\
\hline $\begin{array}{l}\text { Factor 5: } \\
\text { Vasomotor }\end{array}$ & 0.70 & 0.900 & $0.819-0.954$ & 5 \\
\hline Factor 6: Physical & 0.84 & 0.827 & $0.683-0.921$ & 4 \\
\hline Total & 0.84 & 0.935 & 0.8870 .970 & 32 \\
\hline
\end{tabular}




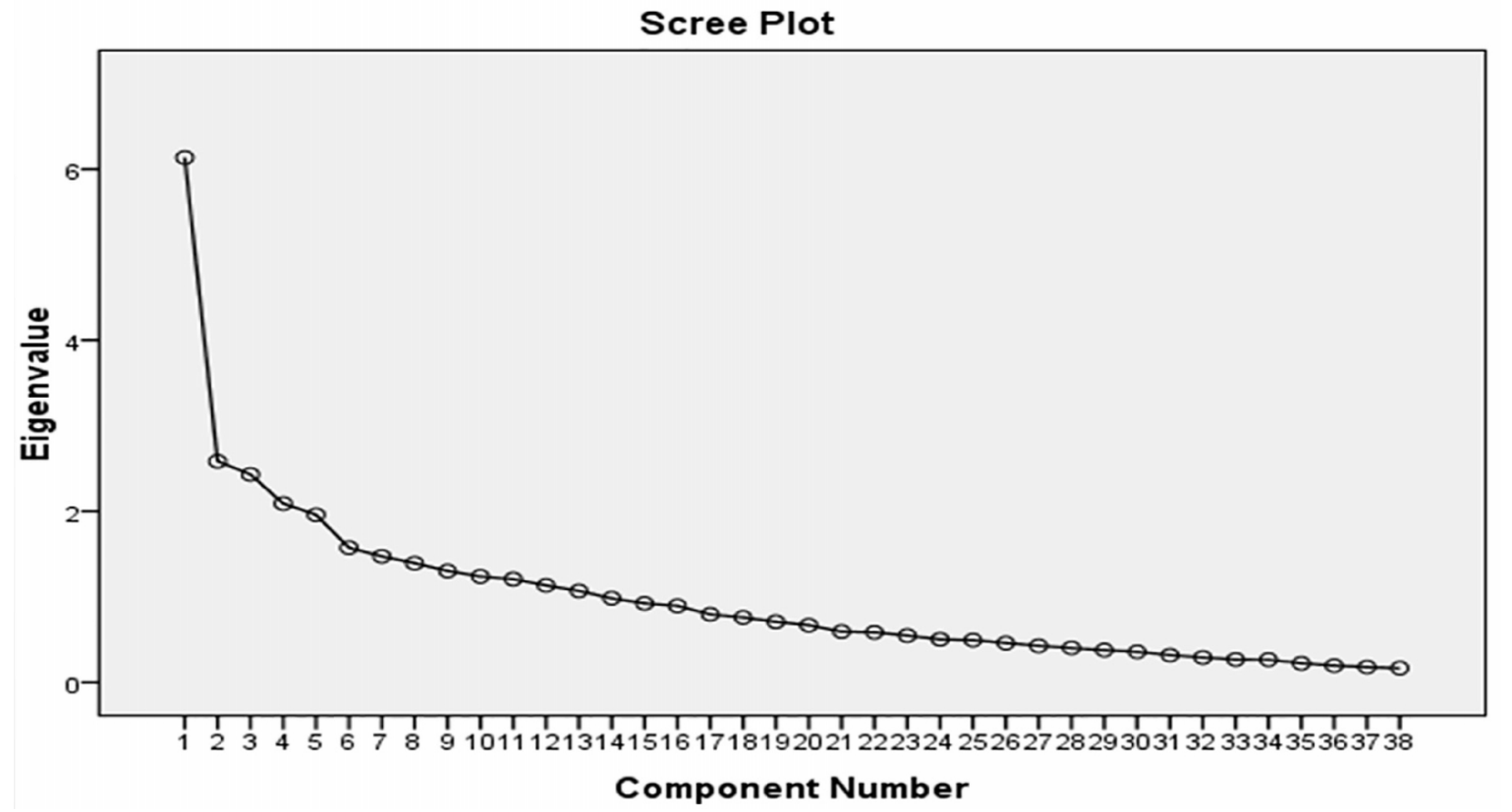

Figure 1. Scree plot figure based on factor analysis to determine the correlation between items

\section{Discussion}

Menopause is an extensive period of hormonal changes in women that actually marks the end of fertility (12). Many symptoms of menopause are stressful. The symptoms or those associated with this period include mood swings, sleep disturbances, urinary incontinence, cognitive changes, physical complaints, sexual dysfunction and reduced quality of life. Changes due to menopause affect individuals' evaluation of their health. Elderly people who have not faced health problems, show more ability compared to those who have encountered health problems (13). International efforts have been done to measure menopausal associated symptoms and problems, and these led to making alternative questionnaires. Some of them are original and others were translated into different languages. The Persian-making method of commonly used instruments has been used in this context in Iran. But dare to say that this study is the first attempt in the field of localization, a tool to check the health of menopausal women in Iran. However, it can be claimed that this study was the first attempt in the field of instrument localization to check menopause health in Iran, which has extensive aspects and therefore it is not immune to error along with its benefits and advantages, compared with instruments translated to Persian. In this study, quantitative and qualitative methods used to assess face validity and the appropriateness of any question to the subject was considered. Coefficient of internal consistency was assessed by Cronbach's alpha, and showed high internal consistency. These findings indicate that the questions are evaluating a similar concept and structure, and concept distribution cannot be seen in them. Cronbach's alpha was performed to study the homogeneity areas. As can be seen in Table 3, the index showed an acceptable size in the 6 domains (mood, urinary, sexual, genital, vasomotor and physical). The obtained ICC value to compare the results between the two iterations was 0.94 . The reproducibility of this method in this study had an acceptable rate. The main instrument for menopause health contains 14 sections of which factor analysis was performed only for part 13, which was related to menopause symptoms. Capability of factor analysis demonstrated that this instrument's questions can be divided into six factors, and section 13 of the questionnaire affirms menopausal symptoms. The six factors were named as follows: 1. mood, 2. urinary, 3. sexual, 4 . genital, 5. vasomotor, and 6. physical. As mentioned above, various instruments have been designed to measure and evaluate menopause symptoms. One of these instruments is Menopause Rating Scale (MRS) which has been designed to measure the severity of symptoms associated with menopause and assess health-related quality of life in this period. This questionnaire is a standard international scale which examines menopausal symptoms based on conducted studies in three parts of somatic (vasomotor), the psychological, and urogenital. In this form, different questions assessed various symptoms of menopause, and it has been used in many national and international studies (12-14). Studies conducted in Iran have either used researcher-made questionnaires or the MRS questionnaire to assess menopausal symptoms, and studies in the country validated the questionnaire and it was estimated to have a correlation coefficient of $0.82(15,16)$. The prevalent symptoms of this period included vasomotor aspects, and physical, mental, and sexual complaints and studies showed that such symptoms are different in various ethnic 
groups, and a number of studies reported that the physical and mental menopause symptoms were lower among Asian women $(17,18)$. For this reason, the present study was conducted to localize a menopausal health questionnaire which involves broader dimensions of health in this period, and present a standard and more comprehensive tool to complete more accurate surveys in the country, and conduct a more complete review of the health status of women in the country. One limitation of the present study was the low rate of Cronbach's alpha and factor loadings for factor 2 (urinary) and factor 6 (physical) in the exploratory factor analysis and it was probably due to the low number of questions in that area. In future studies, there will be brighter factor structure and higher alpha coefficients if items are rewritten, in order to make them more compatible with the Iranian culture.

\section{Conclusions}

The study results showed that the instrument had an acceptable content and construct validity as well as an acceptable internal consistency reliability. In addition, the Menopausal Health Questionnaire covered more dimensions in comparison to other instruments. That is, the Persian version of the 14-item Menopausal Health Questionnaire is a valid and reliable instrument, and its 6 factors (section 13: menopausal symptoms) can be also detected in exploratory factor analysis on responses by Persian subjects.

\section{Acknowledgments:}

The authors express their thanks and appreciation to Ms. Angela Bilancini for allowing the authors of the study to localize the questionnaire, as well as the staff of Ali ibn Abi Talib Hospital in Zahedan affiliated with the University of Medical Sciences, and those who completed the questionnaires due to good cooperation in the implementation of this research study.

\section{Conflict of Interest:}

There is no conflict of interest to be declared.

\section{Authors' contributions:}

All authors contributed to this project and article equally. All authors read and approved the final manuscript.

\section{References:}

1) Mirabi P, Mojab F. The effect of valerian on hot flashes in menopausal women. Iran J Pharm Res. 2013; 12(1): 217-22. PMID: 24250592, PMCID: PMC3813196.

2) Bauld R, Brown RF. Stress, psychological distress, psychosocial factors, menopause symptoms and physical health in women. Maturitas. 2009; 62(2): 160-5. doi: 10.1016/j.maturitas.2008.12.004. PMID: 19167176.

3) Bachmann G, Bobula J, Mirkin S. Effects of bazedoxifene/conjugated estrogens on quality of life in postmenopausal women with symptoms of vulvar/vaginal atrophy. Climacteric. 2010; 13(2): 132-40. doi: 10.3109/13697130903305627. PMID: 19863455.

4) Fritz MA, Speroff L. Clinical gynecologic endocrinology and infertility. Ghotbi R, Ghazi B, Jahani MD. Tehran, Golban Pub. (Persian). 2011.

5) Ogwumike OO, Kaka B, Adegbemigun O, Abiona T. Health-related and socio-demographic correlates of physical activity level amongst urban menopausal women in Nigeria. Maturitas. 2012; 73(4): 349-53. doi: 10.1016/j.maturitas.2012.09.010, PMID: 23063022.

6) Rapkin AJ. Vasomotor symptoms in menopause: physiologic condition and central nervous system approaches to treatment. Am J Obstet Gynecol. 2007; 196(2): 97-106. doi: 10.1016/j.ajog.2006.05.056. PMID: 17306645.

7) Duché L, Ringa V, Melchior M, Varnoux N, Piault S, Zins M, et al. Hot flushes, common symptoms, and social relations among middle-aged nonmenopausal French women in the GAZEL cohort. Menopause. 2006; 13(4): 592-9. doi: 10.1097/01.gme.0000227329.41458.86. PMID: 16837881.

8) Young LE, James AD, Cunningham SL. Lone motherhood and risk for cardiovascular disease: The National Population Health Survey (NPHS), 1998-99. Can J Public Health. 2004; 95(5): 329-35. PMID: 15490920.

9) Jones EG, Mallinson RK, Phillips L, Kang Y. Challenges in language, culture, and modality: Translating English measures into American Sign Language. Nurs Res. 2006; 55(2): 75-81. PMID: 16601619.

10) Mohammadi F, Kaldi A. Self-efficacy and caregiver strain in Alzheimer's caregivers in the city of Tehran. Middle East Journal of Age and Ageing. 2006; 3(3): 8-12. 
11) Yazdikhah M, Keshavarz M, Merghati khoee A, Hosseini F. Effect of a structured educational program with style support group of postmenopausal women Quality. Iranian Journal of Medical Education. 2011; 11: 984-94.

12) Chen Y, Lin SQ, Wei $Y$, Gao HL, Wang SH, Wu ZL. Impact of menopause on quality of life in community-based women in China. Menopause. 2008; 15(1): 144-9. doi: 10.1097/gme.0b013e318115150e. PMID: 18030176.

13) Schneider HP, Maclennan AH, Feeny D. Assessment of health-related quality of life in menopause and aging. Climacteric. 2008; 11(2): 93-107. doi: 10.1080/13697130801936739. PMID: 18365853.

14) Polit DF, Beck CT, Owen SV. Is the CVI an acceptable indicator of content validity? Appraisal and recommendations. Res Nurs Health. 2007; 30(4): 459-67. doi: 10.1002/nur.20199. PMID: 17654487.

15) Chuni N, Sreeramareddy CT. Frequency of symptoms, determinants of severe symptoms, validity of and cut-off score for Menopause Rating Scale (MRS) as a screening tool: a cross-sectional survey among midlife Nepalese women. BMC women's health. 2011; 11: 30. doi: 10.1186/1472-6874-11-30. PMID: 21672198, PMCID: PMC3126771.

16) Ghasemi A, Rezaee A, Khatibi A, Jahanlu A. Efficacy of omega-3 on hot flush in perimenopausal women versus placebo. Medical Science Journal of Islamic Azad Univesity-Tehran Medical Branch. 2012; 22(3): 221-5.

17) Rahman SA, Zainudin SR, Mun VL. Assessment of menopausal symptoms using modified Menopause Rating Scale (MRS) among middle age women in Kuching, Sarawak, Malaysia. Asia Pac Fam Med. 2010; 9(1): 5. doi: 10.1186/1447-056X-9-5. PMID: 20175928, PMCID: PMC2834580.

18) Waidyasekera H, Wijewardena K, Lindmark G, Naessen T. Menopausal symptoms and quality of life during the menopausal transition in Sri Lankan women. Menopause. 2009; 16(1): 164-70. doi: 10.1097/gme.0b013e31817a8abd. PMID: 18703984. 